\title{
Interleukin-13 Is Unlucky for Allergy Sufferers
}

Markus M. Xie, Alexander L. Dent

Department of Microbiology and Immunology, Indiana University School of Medicine, Indianapolis, IN, USA

\begin{abstract}
Anaphylaxis, a life-threatening allergic reaction, is dependent on high affinity allergenspecific IgE. Gowthaman et al. now show that a new interleukin (IL)-13-expressing T helper cell subset specifically promotes high-affinity lgE responses. The discovery of $T$ follicular helper (Tfh)13 cells defines potential new targets for allergy therapies.
\end{abstract}

Keywords: allergy, Tfh13, anaphylaxis

This is the author's manuscript of the article published in final edited form as:

Xie, M. M., \& Dent, A. L. (2019). Interleukin-13 Is Unlucky for Allergy Sufferers. Trends in Pharmacological Sciences, 40(10), 714-716. https://doi.org/10.1016/j.tips.2019.08.007 
The increase in atopic and allergic diseases over the past 30-40 years is of epidemic proportions and these diseases affect millions of people in the USA 1 , $\underline{2}$. Allergic reactions can lead to anaphylaxis, a severe and life-threatening form of allergic immune response. Anaphylaxis is a systemic inflammatory response that results in a dramatic loss of blood pressure and can lead to organ failure. Food allergies, especially to peanut proteins, often involve anaphylactic reactions [3].

During the priming phase of an allergic immune reaction, Interleukin-4 (IL-4) is produced by $T$ helper (Th) cells that promote the production of antigen-specific IgE antibody from $B$ cells. Most IgE then binds strongly to Fce receptors on mast cells. IgE with low affinity for the allergen protein is not sufficient to fully activate mast cells and cannot provoke anaphylaxis in the presence of allergen (Figure 1A). However, the interaction of allergen proteins with high-affinity allergen-specific $\operatorname{lgE}$ on the mast cell surface causes crosslinking of the FcE receptors, triggering a signaling cascade that leads to mast cell degranulation and release of preformed inflammatory mediators stored in cytoplasmic granules, including histamine, proteases, cytokines, and chemokines (Figure 1B) [4]. Release of these inflammatory mediators in a systemic response leads to the severe allergic reaction of anaphylaxis.

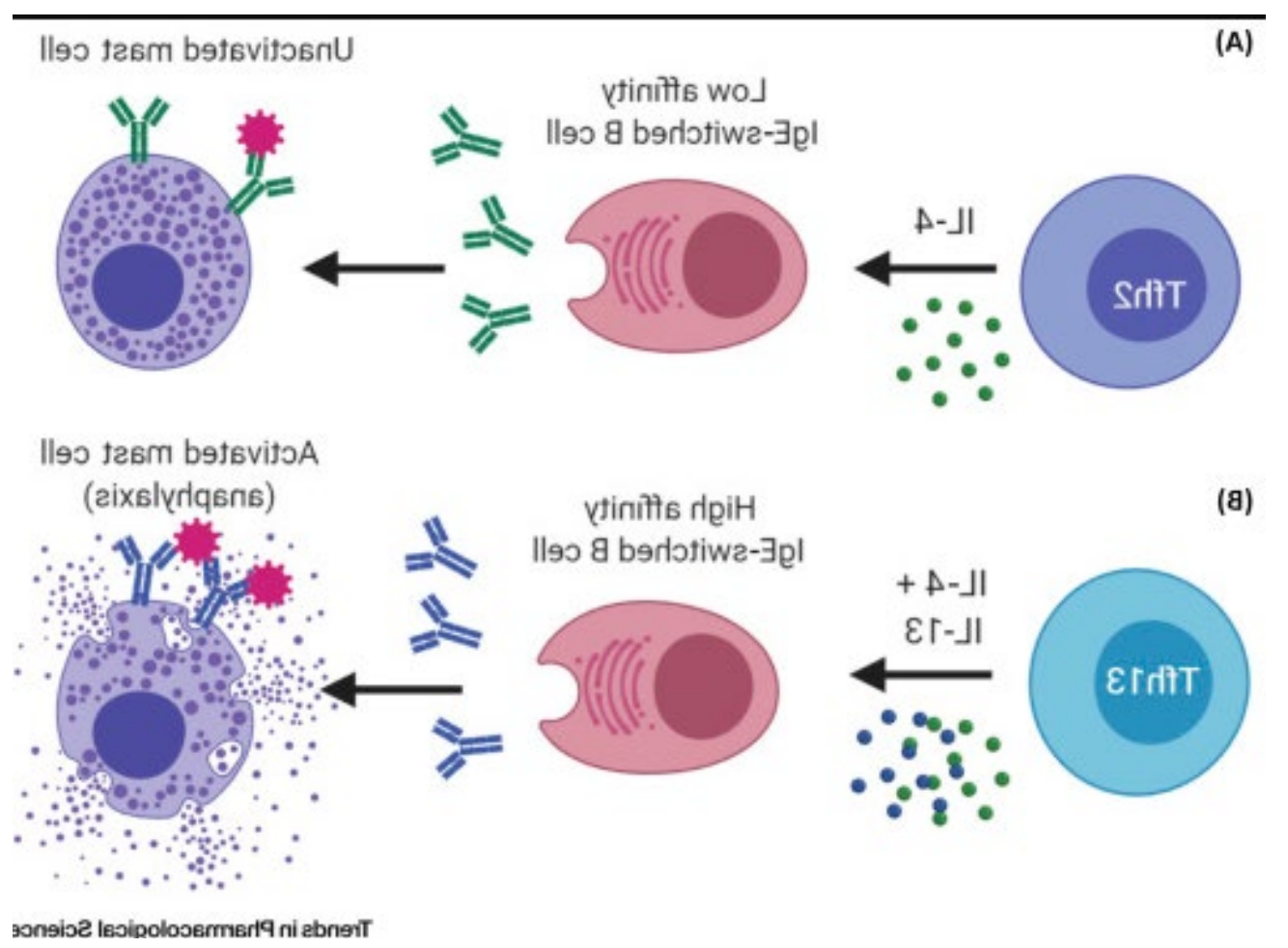


Figure 1. Tfh13 Cells Help to Promote Anaphylaxis through Production of High-Affinity $\lg \mathrm{E}$.

Gowthaman et al. show that conventional Tfh2 cells help B cells produce low-affinity lgE via secretion of IL-4 (A), whereas Tfh13 cells help B cells produce high-affinity IgE via secretion of IL-4 and IL-13 (B). IgE binds to mast cells to mediate hypersensitivity reactions. Low-affinity lgE on mast cells provokes no response when exposed to antigen (red star), whereas crosslinking of high-affinity lgE by antigen on mast cells can induce degranulation and potentially anaphylaxis. This figure was created using BioRender (https://biorender.com/). Abbreviations: IL, interleukin; Tfh, T follicular helper.

Recent studies have revealed that specialized IL-4-secreting T follicular helper (Tfh) cells are essential for the development of antigen-specific $\lg E$ from activated $B$ cells within a cellular structure found in lymphoid tissues called the germinal center [5]. The discovery that Tfh cells control IgE responses has expanded our knowledge of mechanisms that control the development of allergies. However, a crucial aspect of the anaphylactic response is that it is dependent on the development of high-affinity $\lg E$ specific for the allergen [6] and despite the clinical significance of anaphylaxis, the question of how highaffinity, allergen-specific IgE develops in the germinal center has been unclear. Now, investigators from Yale University and the Jackson Laboratory for Genomic Medicine led by Stephanie Eisenbarth have discovered a distinctive IL-13-producing Tfh cell type that is responsible for the induction of high-affinity $\operatorname{lgE}$ that can promote anaphylactic responses [7]. This finding sheds new light onto the diagnosis and potentially the treatment of life-threatening allergies.

It is known that patients with defects in the gene dedicator of cytokinesis 8 (DOCK8) are typically afflicted with hyper-IgE syndrome (HIES), severe allergies and asthma. Gowthaman et al. began their study by characterizing allergic responses in mice that were bred with DOCK8 deficiency in T cells (T-DOCK $\left.{ }^{-1-}\right)$. They observed that an HIES phenotype was found in T-DOCK $8^{--}$mice after type 1 immunization; an immune challenge which should not normally elicit the production of IgE. These results indicated that the HIES phenotype of DOCK8 immunodeficiency syndrome was a T cell-intrinsic effect. Next, using a passive anaphylaxis assay, the authors demonstrated that $\lg E$ from $T$ - 
DOCK ${ }^{-/-}$mice could induce anaphylaxis. However, the frequency and total number of Tfh cells that are required for IgE production, were comparable in T-DOCK ${ }^{-1-}$ and wildtype (WT) mice. This led the authors to analyze the Tfh cells in T-DOCK8--- mice in more detail, and in so doing, they identified a new Tfh cell subset, termed Tfh13 cells, that expressed the cytokine IL-13. The production of IL-13 is classically associated with another subset of Th cells made during allergic immune responses, Th2 cells. Unlike Tfh cells, Th2 cells act outside of the germinal center. Gowthaman et al. found Tfh13 cells in nonmutant WT mice that were induced to have either airway allergy or food allergy, thus linking Tfh13 cell development with allergic disease. Importantly, the development of Tfh13 cells coincided with high levels of high-affinity IgE that could mediate anaphylaxis in WT mice.

Using single-cell RNA sequencing, the authors showed that Tfh 13 cells are a unique cell type significantly different from both Tfh and Th2 cells, but Tfh13 cells also share characteristics of both Th2 and Tfh cells. Unlike conventional Tfh cells, Tfh13 cells express the master transcription factor of Th2 cells, Gata3, and produce the three canonical Th2 cell cytokines IL-4, IL-5, and IL-13. Tfh13 cells also express Bcl6, the master transcription factor for Tfh cells, which represses Gata3 and Th2 cytokine gene transcription [8]. Tfh13 cells appear to maintain a balance between expressing enough Bcl6 to promote the Tfh phenotype and enough Gata3 to transcribe Th2 cytokines. However, an important unresolved question remains as to how Tfh13 cells develop. Do Tfh13 cells differentiate from Th2 cells, from conventional Th cells under strong Th2 polarization conditions, or directly from naïve CD4 T cells?

Gowthaman et al. showed that Tfh13 cells were essential for the production of highaffinity IgE and suggested that IL-4 produced by a subset of Th cells called Tfh2 cells mainly contributed to the production of low-affinity IgE (Figure $1 A$ ). Further work showed that IL-13 produced by Tfh13 cells was indispensable for anaphylactic IgE production (Figure 1B). Gowthaman et al. were also able to transfer their findings into humans, as they found a significantly higher frequency of Tfh 13 cells among circulating Tfh cells from patients with high levels of peanut-specific lgE. These findings helped to resolve a longstanding discrepancy between human and mouse immunology, where IL-13 had been 
known to drive IgE switching in human B cells but not in mouse B cells. Further supporting a role for IL-13, Gowthaman et al. demonstrated that a subset of activated mouse B cells in the germinal center expressed the IL-13 receptor and could be induced to switch to $\operatorname{lgE}$ in the presence of IL-13.

Gowthaman et al. also observed that high-affinity $\lg \mathrm{E}$ was significantly higher in TDOCK $8^{--}$mice, while high-affinity lgG1 was lower compared to that in the control group. As a recent study has shown that high-affinity-IgE-producing plasma cells can differentiate from $\operatorname{lgG} 1^{+}$memory $B$ cells upon re-exposure to allergens [9], the loss of high-affinity lgG1 in T-DOCK8 ${ }^{-/-}$mice indicates that Tfh 13 cells induce a major population of $\operatorname{lgG} 1^{+} B$ cells switching to IgE-secreting $B$ cells. A key question is whether IL-13 produced by Tfh 13 cells helps IgG1 ${ }^{+}$memory $B$ cells switch to IgE-secreting plasma cells. However, whether the switch to IgE occurs from high-affinity $\lg G 1$ memory cells was not addressed. If $\mathrm{IgG} 1^{+}$memory $B$ cells display enriched expression of IL-13 receptor, it could provide a mechanism for this selective response.

Intriguingly, Gowthaman et al. showed that helminth infections could not induce Tfh13 cells or high-affinity lgE, although strong Th2 responses developed. Thus, Th2 cell responses do not always correlate with Tfh13 cell responses. What are the mechanisms for why helminth infections are different from allergies in terms of induction of Tfh13 cells? Solving this question can help us better understand allergies and may also help to develop specific therapeutics for different Th2-mediated diseases such as asthma and atopic dermatitis.

Currently, the only treatment that can prevent the mast cell degranulation response that leads to an anaphylactic shock is nonspecific IgE depletion therapy using omalizumab, a monoclonal antibody that inhibits IgE from binding to mast cells [10]. Treatments that could specifically inhibit the generation of high-affinity IgE could provide an alternative therapeutic strategy to block severe allergic responses and anaphylaxis. Since Tfh13 cells express high levels of GATA3, the use of a GATA3 DNAzyme treatment to downregulate Tfh13 cells and high-affinity lgE may have therapeutic benefit for severe allergies. Gowthaman et al. note that clinical trials of a GATA3 DNAzyme have efficacy in reducing eosinophilia, but it is unknown if this drug could affect Tfh13 or lgE responses. 
Ultimately, understanding the cellular mechanisms for how B cells are stimulated to produce high-affinity lgE in atopic diseases is a clinically important area of immunology research. Eisenbarth and colleagues have now made a major breakthrough on this important question, revealing how a new T cell population, Tfh13 cells, is critical for highaffinity IgE development. These findings lay the groundwork for a deeper understanding of the regulation of high-affinity IgE and anaphylaxis, and provide the basis for precision immunotherapy of atopic diseases.

\section{References}

1. Sicherer, S. H., \& Sampson, H. A. (2007). Peanut allergy: Emerging concepts and approaches for an apparent epidemic. Journal of Allergy and Clinical Immunology, 120(3), 491-503. https://doi.org/10.1016/j.jaci.2007.07.015

2. Asher, M. I., Montefort, S., Björkstén, B., Lai, C. K., Strachan, D. P., Weiland, S. K., \& Williams, H. (2006). Worldwide time trends in the prevalence of symptoms of asthma, allergic rhinoconjunctivitis, and eczema in childhood: ISAAC Phases One and Three repeat multicountry cross-sectional surveys. The Lancet, 368(9537), 733-743. https://doi.org/10.1016/S0140-6736(06)69283-0

3. Iweala, O. I., \& Burks, A. W. (2016). Food Allergy: Our Evolving Understanding of Its Pathogenesis, Prevention, and Treatment. Current Allergy and Asthma Reports, 16(5), 37. https://doi.org/10.1007/s11882-016-0616-7

4. Blank, U., \& Rivera, J. (2006). Assays for Regulated Exocytosis of Mast Cell Granules. Current Protocols in Cell Biology, 32(1), 15.11.1-15.11.18. https://doi.org/10.1002/0471143030.cb1511s32

5. Kobayashi, T., lijima, K., Dent, A. L., \& Kita, H. (2017). Follicular helper T cells mediate IgE antibody response to airborne allergens. Journal of Allergy and Clinical Immunology, 139(1), 300-313.e7. https://doi.org/10.1016/j.jaci.2016.04.021

6. Xiong, H., Dolpady, J., Wabl, M., Curotto de Lafaille, M. A., \& Lafaille, J. J. (2012). Sequential class switching is required for the generation of high affinity IgE antibodies. Journal of Experimental Medicine, 209(2), 353-364. https://doi.org/10.1084/jem.20111941 
7. U. Gowthaman, et al. Identification of a $T$ follicular helper cell subset that drives anaphylactic IgE Science (2019) Published online August 1, 2019. https://doi.org/10.1126/science.aaw6433

8. Vinuesa, C. G., Linterman, M. A., Yu, D., \& MacLennan, I. C. M. (2016). Follicular Helper $\mathrm{T}$ Cells. Annual Review of Immunology, 34(1), 335-368. https://doi.org/10.1146/annurev-immunol-041015-055605

9. He, J.-S., Subramaniam, S., Narang, V., Srinivasan, K., Saunders, S. P., Carbajo, D., Wen-Shan, T., Hidayah Hamadee, N., Lum, J., Lee, A., Chen, J., Poidinger, M., Zolezzi, F., Lafaille, J. J., \& Curotto de Lafaille, M. A. (2017). IgG1 memory B cells keep the memory of $\operatorname{lgE}$ responses. Nature Communications, 8(1), 641. https://doi.org/10.1038/s41467-017-00723-0

10.Kawakami, T., \& Blank, U. (2016). From IgE to Omalizumab. The Journal of Immunology, 197(11), 4187-4192. https://doi.org/10.4049/jimmunol.1601476 